\title{
Noninvasive stimulation of prefrontal cortex strengthens existing episodic memories and reduces forgetting in the elderly
}

\section{Marco Sandrini ${ }^{1,2 *}$, Michela Brambilla $^{3}$, Rosa Manenti ${ }^{3}$, Sandra Rosini ${ }^{3}$, Leonardo G. Cohen ${ }^{1}$ and Maria Cotelli ${ }^{3}$}

\author{
'Human Cortical Physiology and Neurorehabilitation Section, National Institute of Neurological Disorders and Stroke, National Institutes of Health, Bethesda, \\ MD, USA \\ ${ }^{2}$ Center for Neuroscience and Regenerative Medicine, Uniformed Services University of Health Sciences, Bethesda, MD, USA \\ ${ }^{3}$ Neuropsychology Unit, IRCCS Istituto Centro San Giovanni di Dio Fatebenefratelli, Brescia, Italy
}

Edited by:

David Bartrés-Faz, University of

Barcelona, Spain

Reviewed by:

Amir Homayoun Javadi, University College London, UK

Robert Blumenfeld, University of

California, Irvine, USA

\section{*Correspondence:}

Marco Sandrini, Human Cortical

Physiology and Neurorehabilitation

Section, National Institute of

Neurological Disorders and Stroke,

National Institutes of Health,

Building 10, Room 7D52, Bethesda,

MD 20892, USA

e-mail: marco.sandrini@nih.gov
Memory consolidation is a dynamic process. Reactivation of consolidated memories by a reminder triggers reconsolidation, a time-limited period during which existing memories can be modified (i.e., weakened or strengthened). Episodic memory refers to our ability to recall specific past events about what happened, including where and when. Difficulties in this form of long-term memory commonly occur in healthy aging. Because episodic memory is critical for daily life functioning, the development of effective interventions to reduce memory loss in elderly individuals is of great importance. Previous studies in young adults showed that the dorsolateral prefrontal cortex (DLPFC) plays a causal role in strengthening of verbal episodic memories through reconsolidation. The aim of the present study was to explore the extent to which facilitatory transcranial direct current stimulation (anodal tDCS) over the left DLPFC would strengthen existing episodic memories through reconsolidation in elderly individuals. On Day 1, older adults learned a list of 20 words. On Day 2 ( $24 \mathrm{~h}$ later), they received a reminder or not, and after 10 min tDCS was applied over the left DLPFC. Memory recall was tested on Day 3 (48 h later) and Day 30 (1 month later). Surprisingly, anodal tDCS over the left DLPFC (i.e., with or without the reminder) strengthened existing verbal episodic memories and reduced forgetting compared to sham stimulation. These results provide a framework for testing the hypothesis that facilitatory tDCS of left DLPFC might strengthen existing episodic memories and reduce memory loss in older adults with amnestic mild cognitive impairment.

Keywords: tDCS, aging, reconsolidation, episodic memory, prefrontal cortex, memory, enhancement, forgetting

\section{INTRODUCTION}

Episodic memory refers to the recollection of personal experiences that contain information on what has happened and also where and when these events took place (Tulving, 1983). This form of long-term memory displays the largest degree of age-related decline (Ronnlund et al., 2005; Vestergren and Nilsson, 2010). Older adults, for example, have more difficulty recalling what they had for breakfast than do younger adults. Studies in the cognitive neuroscience of aging have begun to link declining episodic memory to neurochemical, structural and functional brain changes (Grady and Craik, 2000; Cabeza and Lennartson, 2005; Daselaar and Cabeza, 2008; Nyberg et al., 2012; Sala-Llonch et al., 2014). Because this memory is critical for daily life functioning and its decline is accelerated in conditions like amnestic mild cognitive impairment (aMCI) and Alzheimer's disease (AD), the development of effective interventions to reduce memory loss in elderly individuals and in patients with $\mathrm{aMCI}$ or $\mathrm{AD}$ is of great importance
(Buschert et al., 2011; Alberini and Chen, 2012; Cotelli et al., 2012).

For more than a century, it was generally assumed that memories are unstable (i.e., susceptible to interference) for a limitedtime after encoding, but as time passes, memories stabilize and become resistant to interference (McGaugh, 2000). However, this classical consolidation view has been challenged over the past 15 years by accumulating evidence showing that consolidated memories can re-enter unstable states when they are reactivated during retrieval or by a reminder cue and need to consolidate again in order to persist over longer periods of time (Nader et al., 2000a,b; Nader and Hardt, 2009; Dudai, 2012). Thus, the concept of reconsolidation assumes that memories are not consolidated once and forever, challenging the view that stability characterizes consolidated memories (Alberini and Ledoux, 2013). Indeed, memory reactivation triggers reconsolidation, a time-limited period during which the existing memory traces are vulnerable to modifications (Nadel et al., 2012; Alberini and 
Ledoux, 2013; Schwabe et al., 2014). There is evidence that existing episodic memories can be strengthened (Coccoz et al., 2011, 2013; Finn and Roediger, 2011; Forcato et al., 2011; Javadi and Cheng, 2013; Rodriguez et al., 2013; Sandrini et al., 2013; Bos et al., 2014), weakened/disrupted (Forcato et al., 2007; Strange et al., 2010; Chan and LaPaglia, 2013; Schwabe et al., 2013; Kroes et al., 2014), or updated by the inclusion of new information (Hupbach et al., 2007, 2008, 2009) through reconsolidation.

Clinical studies have shown that episodic memory is primarily dependent on the integrity of the medial temporal lobe. Functional neuroimaging (Fletcher and Henson, 2001; Simons and Spiers, 2003) and noninvasive brain stimulation (NIBS) studies (Manenti et al., 2012; Brem et al., 2013; Manenti et al., 2013) have also emphasized the contribution of the lateral prefrontal cortex (PFC) to episodic memory processes. In addition, evidence from neuroimaging, neurophysiology and computational modeling highlights the importance of interactions between these brain regions for memory function (Simons and Spiers, 2003).

NIBS techniques (Sandrini et al., 2011; Dayan et al., 2013), such as transcranial magnetic stimulation (TMS) and trascranial direct current stimulation (tDCS), have been used for two purposes in the study of memory function: (1) to test the causal relationship between activity in specific cortical regions and memory function; and (2) to test the general hypothesis that NIBS could modulate memory formation and learning, an issue of obvious relevance for memory research and neurorehabilitation (Zimerman and Hummel, 2010; Sandrini and Cohen, 2013, 2014).

Recent NIBS studies have showed that the dorsolateral PFC (DLPFC) plays a causal role in strengthening of verbal episodic memories through reconsolidation in healthy young adults (Javadi and Cheng, 2013; Sandrini et al., 2013).

Sandrini et al. (2013) applied repetitive TMS (rTMS) over the right DLPFC, a region critically involved in retrieval of verbal (Sandrini et al., 2003; Gagnon et al., 2010, 2011; Manenti et al., 2010) and nonverbal (Rossi et al., 2001, 2004; Gagnon et al., 2010, 2011) episodic memories. Participants learned a list of words on Day 1. On Day 2 (24h after the learning session), in a group of subjects existing memories were reactivated by a spatialcontextual reminder cue (i.e., same experimental room of Day 1) and $10 \mathrm{~min}$ later $1 \mathrm{~Hz}$ rTMS was applied to the right DLPFC. To determine whether the rTMS effect was specific to memory reactivation and relied on right PFC function, the authors designed two control groups. First, to determine whether the rTMS effect was specific to memory reactivation, they applied rTMS over the right DLPFC without memory reactivation (i.e., different experimental room), a behavioral manipulation previously successfully done in human reconsolidation studies (Hupbach et al., 2007, 2008). Second, to determine whether the rTMS effect was topographically specific, they applied rTMS over the vertex (i.e., control site) (Censor et al., 2010; Sandrini et al., 2011) after memory reactivation. Memory recall was tested on Day 3 ( $48 \mathrm{~h}$ after the learning session). The results demonstrated that rTMS over the right DLPFC after memory reactivation strengthened existing verbal episodic memories, an effect indicated by enhanced memory recall $24 \mathrm{~h}$ later $(73 \%)$ compared to control conditions (DLPFC without memory reactivation $=56.3 \%$; vertex-rTMS $=56.6 \%)$.
In a similar study, Javadi and Cheng (2013) applied tDCS over the left DLPFC, a region critically involved in encoding of verbal (Sandrini et al., 2003; Gagnon et al., 2010, 2011; Javadi and Walsh, 2012) and nonverbal (Rossi et al., 2001, 2004; Gagnon et al., 2010, 2011) episodic memories. Participants memorized words in the first session. Three hours later, in the second session, participants in the reconsolidation group underwent $\mathrm{tDCS}$ while the existing memories were reactivated during retrieval (old-new recognition task). The final session was scheduled to occur $5 \mathrm{~h}$ after the stimulation session. The old-new word recognition task was employed here as a measure of memory performance. The results showed that anodal tDCS enhanced episodic memory recognition compared to cathodal and sham stimulation. In order to test whether the reactivation of the existing memories was crucial for the enhancing effects of anodal tDCS, a control group did not perform the recognition task in the second session but still underwent stimulation (i.e., no reactivation condition). Contrary to the reconsolidation group, anodal stimulation did not enhance the memory performance for the control group. This result suggests that anodal tDCS over the left DLPFC enhances the reconsolidation of long-term memory only when the existing memories have been reactivated during retrieval.

Thus, the findings of these studies (Javadi and Cheng, 2013; Sandrini et al., 2013) show that noninvasive stimulation of DLPFC during reconsolidation has the potential to serve as a novel strategy to induce long-lasting memory enhancements in individuals with episodic memory decline.

In addition, tDCS over the DLPFC during retrieval enhanced episodic memory in healthy young and older adults (Manenti et al., 2013). In this study, each participant underwent two sessions of anodal tDCS (left and right) and one session of sham stimulation. The results showed that anodal tDCS applied over the left and right DLPFC induced better recognition performance in young subjects compared to sham stimulation. However, only anodal tDCS applied over the left DLPFC enhanced memory retrieval in older subjects.

Using Sandrini et al.s paradigm (Sandrini et al., 2013) with an additional memory recall session after 30 days, the aim of the present study was to explore the extent to which anodal tDCS over the left DLPFC would strengthen existing episodic memories through reconsolidation in elderly individuals.

\section{MATERIALS AND METHODS PARTICIPANTS}

Thirty-six healthy older individuals (24 females and 12 males; mean age $=67.17 \pm 3.68$ years; mean education $=12.05 \pm$ 4.40 years) took part in the experiment. All of the subjects had normal or corrected-to-normal vision and were native Italian speakers (see Table 1 for demographic details). The 36 enrolled participants were randomly assigned to one of three experimental groups: Anodal-R (anodal tDCS-Reminder); Anodal-NR (anodal tDCS-No Reminder); Sham-R (sham tDCS-Reminder). tDCS was applied over the left DLPFC as in previous verbal episodic memory studies in young (Javadi and Cheng, 2013; Manenti et al., 2013) and older adults (Manenti et al., 2013).

Participants reported being free of neurological disorders and had no history of seizures. All participants were informed 
Table 1 | Demographic characteristics of older individuals grouped according to the experimental conditions (Anodal-NR; Anodal-R; Sham-R), and Cognitive Reserve Index (CRI-q) and strategies questionnaire scores.

\begin{tabular}{lccc}
\hline & $\begin{array}{c}\text { Anodal-NR } \\
(\boldsymbol{n}=\mathbf{1 2})\end{array}$ & $\begin{array}{c}\text { Anodal-R } \\
(\boldsymbol{n}=\mathbf{1 2})\end{array}$ & $\begin{array}{c}\text { Sham-R } \\
(\boldsymbol{n}=\mathbf{1 2})\end{array}$ \\
\hline Age (years) & $67.5 \pm 2.7$ & $67.6 \pm 4.3$ & $66.4 \pm 4.0$ \\
Education (years) & $11.8 \pm 5.0$ & $11.3 \pm 3.9$ & $13.2 \pm 4.4$ \\
COGNITIVE RESERVE INDEX QUESTIONNAIRE (CRI-q) & \\
CRI-total score & $118.4 \pm 20.7$ & $119.3 \pm 17.0$ & $123.7 \pm 21.9$ \\
CRI-education & $110.1 \pm 15.6$ & $104.8 \pm 10.3$ & $117.2 \pm 12.8$ \\
CRI-working activity & $108.8 \pm 13.7$ & $103.8 \pm 17.3$ & $106.7 \pm 20.2$ \\
CRI-leisure time & $122.7 \pm 28.9$ & $130.0 \pm 22.1$ & $129.7 \pm 22.1$ \\
STRATEGIES QUESTIONNAIRE & & \\
Strategies total score & $8.6 \pm 4.0$ & $6.4 \pm 3.3$ & $7.4 \pm 3.8$
\end{tabular}

about the procedures and the possible risks of tDCS, and written informed consent was obtained after a safety screening. The experimental methods got ethical approval from the local Human Ethics Committee (CEIOC) of Saint John of God Clinical Research Centre, IRCCS Istituto Centro San Giovanni di Dio Fatebenefratelli, Brescia, Italy. Prior to being enrolled in the experiment, older subjects completed a Mini Mental State Examination (MMSE) (Folstein et al., 1975) and a detailed neuropsychological evaluation to verify the absence of any cognitive deficit. A pathological score in one or more of the tests was an exclusion criterion. The neuropsychological test battery included measures used to assess non-verbal reasoning (Raven's Colored Progressive Matrices), verbal fluency (phonemic and semantic), visuo-spatial capacity (Rey-Osterrieth Complex Figure, Copy), upper-limb apraxia (De Renzi et al., 1980), attention and executive functions (Trail Making Test A and B). Moreover, memory was assessed in depth (Story Recall, ReyOsterrieth Complex Figure Recall, Digit Span, Auditory Verbal Learning Test learning and recall). All of the tests were administered and scored according to standard procedures (Lezak et al., 2004). The results of the cognitive assessments are presented in Table 2. In addition, we administered The Cognitive Reserve Index questionnaire (CRIq), which provides a standardized measure of the cognitive reserve accumulated by individuals through their lifespan. The CRIq includes demographic data and items grouped into three sections: education, working activity and leisure time, each of which returns a subscore and compose the total score (Nucci et al., 2012) (see Table 1).

\section{STIMULI}

We selected 20 concrete words from the "Corpus e Lessico di Frequenza dell'Italiano Scritto (CoLFIS)" (Laudanna et al., 1995). The words were balanced according to word length and to variables known to influence memory performance, i.e., word frequency, familiarity and imageability (see Appendix A for details). The selected words were highly imageable and concrete to ensure that participants knew all the words and were able to imagine them.

\section{TASK PROCEDURE AND EXPERIMENTAL DESIGN}

The experiment consisted of four sessions on four different days: Day 1 (learning session), Day 2 (24 h after learning session), Day 3 (48 h after learning session) and Day 30 (1 month after learning session). Participants were informed that they would have to memorize a list of object words and that in the second day they would receive a 15 min session of tDCS. No information was given to participants regarding the third day and the session after 1 month (i.e., recall sessions).

On Day 1 , subjects $(n=36)$ were asked to learn a list of 20 object words (see Appendix A). This procedure was repeated until the participants remembered at least 17 of the 20 words $(85 \%)$ or until a maximum of five learning trials was reached. The experimenter pulled out one item at a time at random (a word written on piece of cardboard) from a white bag. Participants were asked to name each word, to pay close attention so they could remember the words later and to place them in a distinctive blue bag. After all 20 words were placed into a blue bag, the experimenter took away this bag and asked the participants to remember as many words as possible. Before the next learning trial, the words were placed in the white bag again and mixed. The entire learning session took about $20-25 \mathrm{~min}$ to complete.

At the end of this experimental session, all 36 subjects were asked to fill in a "Memory strategies questionnaire." This questionnaire comprised 12 possible strategies that could be used during the task and subjects had to assign a score from 1 to $10(1=$ never, $10=$ always $)$ to each strategy according to how often they had used each strategy during the task. The 12 listed strategies were: (i) to use words' initials, (ii) to create sentences including some of the presented words, (iii) to imagine the pictures corresponding to the presented words, (iv) to repeat the words, (v) to create songs including some of the presented words, (vi) to create rhymes between the displayed words, (vii) to translate the words in a foreign language, (viii) to create associations of words, (ix) to create a brief story including the presented words, (x) to associate each word to a personal event, (xi) to classify each word as easy/difficult, abstract/concrete, positive/negative, etc., (xii) to imagine the words' sound, color, shape, etc. (Manenti et al., 2010).

On Day 2 (24 h after the learning session), the procedure differed for the three experimental groups. For the Anodal- $\mathrm{R}$ and Sham-R groups, the experimenter, who administered the procedure in the same experimental room on Day 1 , showed them the empty blue bag and asked, "Do you remember this blue bag and what we did with it yesterday?" Participants were encouraged to describe the procedure, but were stopped if they started to recall any specific words. On the basis of previous findings showing that the reconsolidation process seems to begin between 3 and $10 \mathrm{~min}$ after memory reactivation (Monfils et al., 2009), subjects received tDCS (anodal or sham) $10 \mathrm{~min}$ after the reminder. There is evidence that existing memories are automatically reactivated if the subjects are in the same experimental room of Day 1 (Hupbach et al., 2008).

For the Anodal-NR group, a new experimenter administered the experimental procedure in a different experimental room. The experimenter only applied anodal tDCS without presenting the 
Table 2 | Neuropsychological assessment of older subjects grouped according to the experimental conditions (Anodal-NR; Anodal-R; Sham-R).

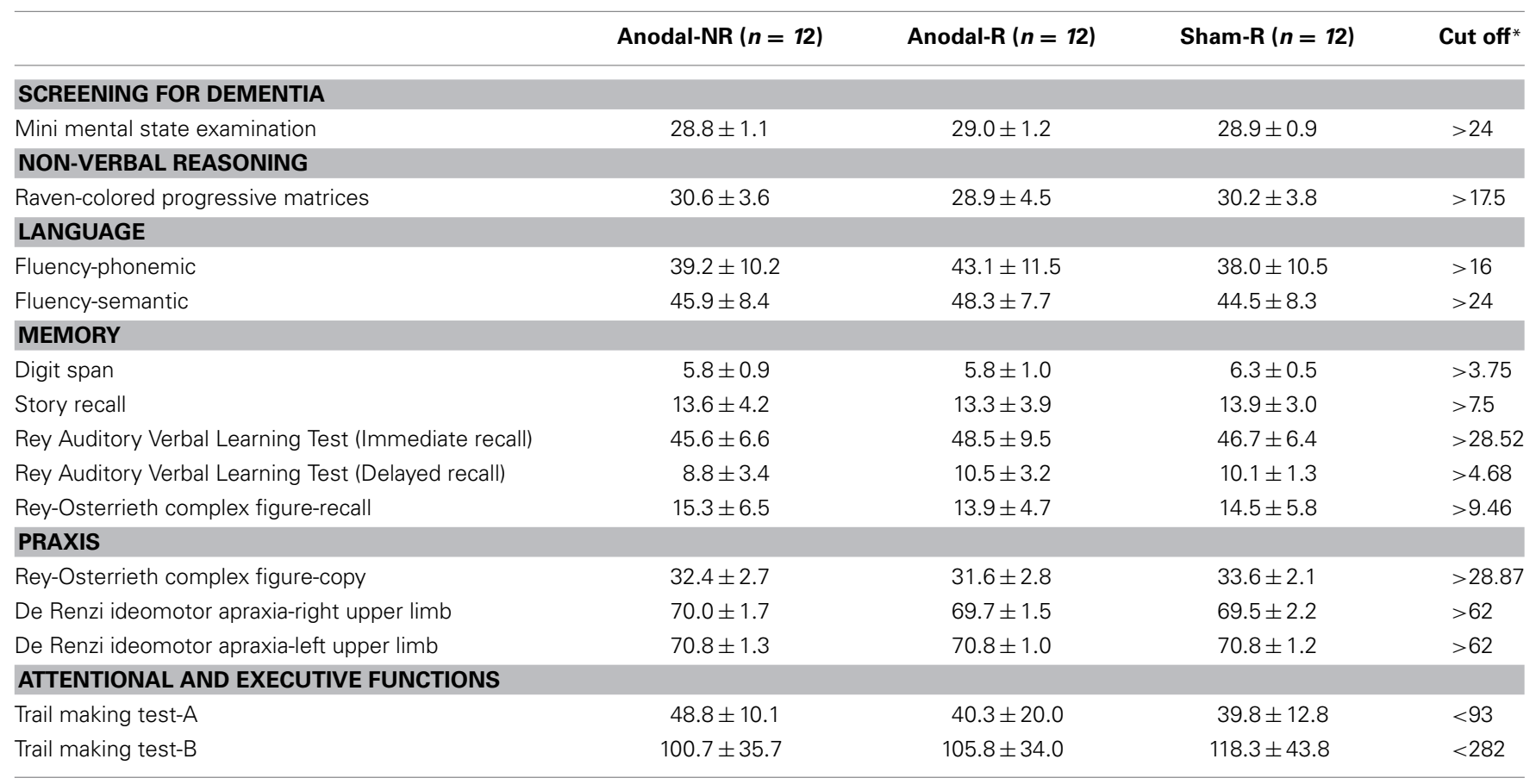

${ }^{*}$ Cut-off scores according to Italian normative data. Raw scores are reported.

blue bag and asking what had happened on Day 1. Day 2 session took on average $30 \mathrm{~min}$ to complete.

On Day 3 ( $48 \mathrm{~h}$ after the learning session), the experimenter asked the participants to recall as many words as possible from Day 1, and the experimenter noted the remembered words. When participants indicated that they could not remember any more words, the experimenter engaged the participants in a figure copying task for about $30 \mathrm{~s}$. The experimenter repeated the recall test by asking the participants to recall the words again. This procedure was repeated for a total of four consecutive recall trials in order to test reliability of recall. The recall session took about 15 min to complete.

On Day 30 (1 month after learning session), the procedure was exactly the same of Day 3.

The experiment design is based on a previous reconsolidation study (Sandrini et al., 2013) and is illustrated in detail in Figure 1.

\section{tDCS PROCEDURE}

tDCS is a safe, portable NIBS technique in which electrical current is directly applied to the head to generate an electrical field that modulates neuronal activity (Nitsche and Paulus, 2000; Nitsche et al., 2008; Dayan et al., 2013). Anodal tDCS has a general facilitation effect and causes membrane depolarization, whereas cathodal tDCS has a general inhibitory effect and causes membrane hyperpolarization.

A battery-driven stimulator (BrainStim, EMS, Bologna, Italy) delivered low, constant current through a pair of saline-soaked sponge electrodes $(7 \times 5 \mathrm{~cm})$. A constant current of $1.5 \mathrm{~mA}$ was applied for $15 \mathrm{~min}$ (with a ramping period of $10 \mathrm{~s}$ at the beginning and end of the stimulation). The current density $\left(0.043 \mathrm{~mA} / \mathrm{cm}^{2}\right)$ was maintained below safety limits (Poreisz et al., 2007). The electrodes were secured using elastic bands, and to reduce contact impedance, an electroconductive gel was applied under the electrodes before the montage. The study was a randomized single-blind experiment: the subjects did not know which stimulation they received, but the experimenter did. Twenty-four participants received anodal (AtDCS- NR and Anodal-R Groups) and 12 subjects received sham (StDCS-R) tDCS stimulation over the left DLPFC, a region critically involved in retrieval of episodic memories in older subjects (Manenti et al., 2013). For anodal stimulation of the left DLPFC, the anode was placed over F3 according to the 10-20 EEG international system for electrode placement, and the cathode was placed over the right supraorbital area (Herwig et al., 2003). In the sham stimulation, the tDCS montage was the same, but the current was turned off $10 \mathrm{~s}$ after the beginning of the stimulation (plus the duration of the fadein $=10 \mathrm{~s}$ ) and was turned on for the last $10 \mathrm{~s}$ of the stimulation period (plus the duration of the fade-out $=10 \mathrm{~s}$ ) ( see Figure 1). Therefore, subjects felt the itching sensations below the electrodes at the beginning and at the end of the stimulation, making this condition indistinguishable from the experimental stimulation (Gandiga et al., 2006). Potential tDCS side effects were assessed with a questionnaire at administered at the end of the stimulation session (Fertonani et al., 2010).

\section{STATISTICAL ANALYSES}

Demographic, neuropsychological variables, tDCS sensations, cognitive reserve and strategy use were compared between the three experimental groups using parametric ( $t$-test) and nonparametric (Kruskal-Wallis test) analyses were measured.

A $3 \times 3$ repeated measures ANOVA was used to analyze the mean percentage of words correctly recalled with one 


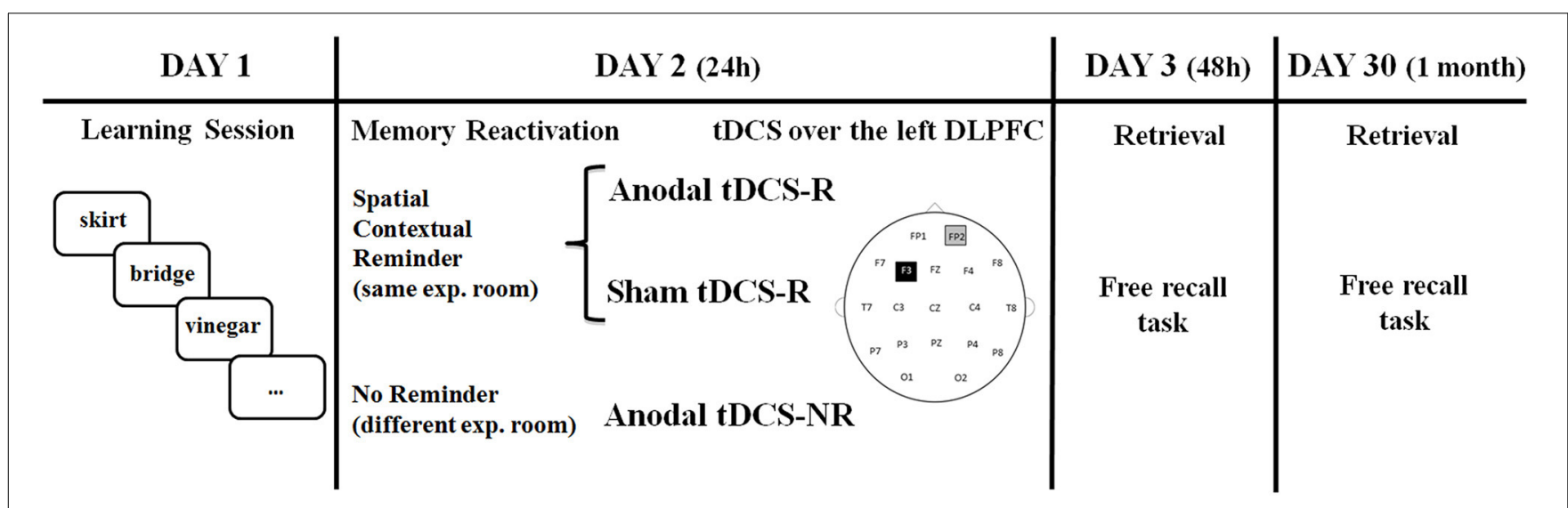

FIGURE 1 | Timeline of experiment. Older adults learned 20 words on Day 1. On Day 2 ( $24 \mathrm{~h}$ later), they received a reminder or not, and after $10 \mathrm{~min}$ tDCS was applied over the left DLPFC. Memory retrieval (free recall) was tested on Day 3 ( $48 \mathrm{~h}$ later) and Day 30 (1 month later).

within-group factor of Time (Day 1, Day 3, and Day 30) and one between-group factor of Group (Anodal-NR, AnodalR, Sham-R). Post-hoc analysis was carried out by Fisher's Least Significant Difference (LSD) tests for evaluating pairwise comparisons among levels of ANOVA significant factors in order to discover which of the comparisons were responsible for rejections in ANOVA test (Hayter, 1986). Statistical analyses were performed using Statistica software (version 10; www.statsoft.com).

\section{RESULTS}

No differences in age $\left[H_{(2)}=1.53, p=0.82\right]$, education $\left[H_{(2)}=2.78, p=0.60\right]$, tDCS sensations $\left[H_{(2)}=4,9, \mathrm{p}=0.30\right]$, strategy use $\left[H_{(4)}=2.92, p=0.57\right]$, and cognitive reserve $\left[H_{(2)}=0.54, p=0.97\right]$ were observed between the experimental groups (see Table 1). Furthermore, no differences in neuropsychological tests were shown in the Table 2 .

Perceptual sensations induced by the anodal tDCS and sham tDCS conditions were assessed with standardized questionnaire developed by Fertonani et al. (2010). Participants were asked to evaluate intensity of several perceptual sensations (i.e., itching, pain, burning, heat, pinching, iron taste, fatigue, effect on performance) through a 5 -point-scale $(0$, none; 1 , mild; 2 , moderate; 3 , considerable; and 4, strong). By interpreting the questionnaire completed by all subjects at the end of each type of stimulation we inferred that all the subjects tolerated well the stimulation and reported only marginal perceptual sensations. Itching and irritation were the most commonly reported perceptual sensations, with light to moderate intensity. Overall, the experienced perceptual sensations started at the beginning of the experiment and did not last long. For each group (real and sham stimulation), the sensations scores reported during anodal tDCS were compared with the sensations reported during the sham tDCS by a single-tailed independent $t$-test. These analyses showed that the anodal stimulations could not be distinguished from the sham $\left[H_{(2)}=4.9, p=0.30\right]$. Hence there are no reasons to reject the single-blinded character of this study on the basis of these results.

\section{TASK RESULTS}

In order to compare the learning rate of the three experimental groups, we recorded how many learning trials (1-6) were necessary for participants to recall at least 17 words (85\%) on Day 1. Participants who recalled $<17$ words during the fifth learning trial were given a score of 6. Participants needed on average 4.9 (SD 1.1) learning trials to reach this criterion (Anodal-R = 5.2 SD 1.0; Anodal-NR $=4.8$ SD 1.3; Sham-R = 4.8 SD 1.0). There were no significant differences between the three groups $(H=0.87, p=$ 0.65 ). Furthermore, participants recalled on average the $79.2 \%$ of the words at the last learning trial (SD 11.7) to reach this criterion (Anodal-R $=81.6 \%$ SD 12.9; Anodal-NR $=77.5 \%$ SD 11.4; Sham- $\mathrm{R}=78.3 \%$ SD 10.9). There were no significant differences between the three groups $(H=1.56, p=0.81)$.

The mean percentage of words correctly recalled was analyzed with ANOVA with "group" (Anodal-R, Anodal-NR, Sham-R) as the between subjects variable and "time" (Day 1, Day 3, and Day 30) as the within-subjects variable.

The analyses showed significant effects for "group" $\left[F_{(2,33)}=\right.$ 4.64, $\left.p=0.02, \eta_{p}^{2}=0.220\right]$, "time" $\left[F_{(2,66)}=73.8, p<0.001\right.$, $\left.\eta_{p}^{2}=0.691\right]$ and the interaction between "group" and "time" $\left[F_{(4,66)}=3.56, p=0.01, \eta_{p}^{2}=0.178\right]$. Based on these results, we were interested in evaluating pairwise comparisons among levels of significant effects. In particular we wanted to highlight the performance accuracy differences from Day 1 to Day 3 and from Day 1 to Day 30 in all groups and compare performance among different groups. Post-hoc Analyses were performed using LSD test and all $p$-values are reported in Table 3.

Considering the group effect, the post-hoc comparisons showed significant differences between Anodal-NR (mean 49.9; SD 21.6) and Sham-R (mean 31.9; SD 17.1) $(p=0.04)$ and between Anodal-R (mean 49.9; SD 24.6) and Sham-R $(p<0.01)$. No difference was found between Anodal-NR and Anodal-R ( $p=0.47$ ) (see Figure 2).

Considering the time effect, significant differences were found between Day 1 and Day $3(p<0.01)$ and between Day 1 and Day $30(p<0.01)$. No difference was found between Day 3 and Day $30(p=0.73)$. 
Table 3 | p-Values of all post-hoc analyses using LSD test.

\begin{tabular}{|c|c|c|c|c|c|c|c|c|c|c|}
\hline & & \multicolumn{3}{|c|}{ Anodal-NR } & \multicolumn{3}{|c|}{ Anodal-R } & \multicolumn{3}{|c|}{ Sham-R } \\
\hline & & Day 1 & Day 3 & Day 30 & Day 1 & Day 3 & Day 30 & Day 1 & Day 3 & Day 30 \\
\hline & Mean & $77.5 \%$ & $49.9 \%$ & $47.2 \%$ & $81.6 \%$ & $49.9 \%$ & $56.3 \%$ & $78.3 \%$ & $31.9 \%$ & $24.7 \%$ \\
\hline & SD & 11.4 & 17.3 & 25.9 & 12.9 & 24.3 & 25.6 & 10.9 & 16.6 & 17.6 \\
\hline \multicolumn{11}{|c|}{ ANODAL-NR } \\
\hline Day 1 & & & $0.000^{*}$ & $0.000^{*}$ & 0.591 & $0.001^{*}$ & $0.007^{*}$ & 0.914 & $0.000^{*}$ & $0.000^{*}$ \\
\hline Day 3 & & $0.000^{*}$ & & 0.648 & $0.000 *$ & 1.000 & 0.413 & $0.000^{*}$ & $0.022 *$ & $0.002 *$ \\
\hline Day 30 & & $0.000^{*}$ & 0.648 & & $0.000 *$ & 0.727 & 0.244 & $0.000^{*}$ & 0.051 & $0.005^{*}$ \\
\hline \multicolumn{11}{|c|}{ ANODAL-R } \\
\hline Day 1 & & 0.591 & $0.000^{*}$ & $0.000 *$ & & $0.000 *$ & $0.000^{*}$ & 0.667 & $0.000^{*}$ & \\
\hline Day 3 & & $0.001^{*}$ & 1.000 & 0.727 & $0.000 *$ & & 0.286 & $0.000 *$ & $0.022 *$ & \\
\hline Day 30 & & $0.007^{*}$ & 0.413 & 0.244 & $0.000 *$ & 0.286 & & $0.005^{*}$ & $0.002 *$ & $0.000 *$ \\
\hline \multicolumn{11}{|c|}{ SHAM-R } \\
\hline Day 1 & & 0.914 & $0.000^{*}$ & $0.000 *$ & 0.667 & $0.000 *$ & $0.005^{*}$ & & $0.000^{*}$ & $0.000^{*}$ \\
\hline Day 3 & & $0.000 *$ & $0.022 *$ & 0.051 & $0.000 *$ & $0.022 *$ & $0.002 *$ & $0.000 *$ & & 0.228 \\
\hline Day 30 & & $0.000 *$ & $0.002 *$ & $0.005^{*}$ & $0.000 *$ & $0.002^{*}$ & $0.000^{*}$ & $0.000^{*}$ & 0.228 & \\
\hline
\end{tabular}

${ }^{*} p<0.05$.

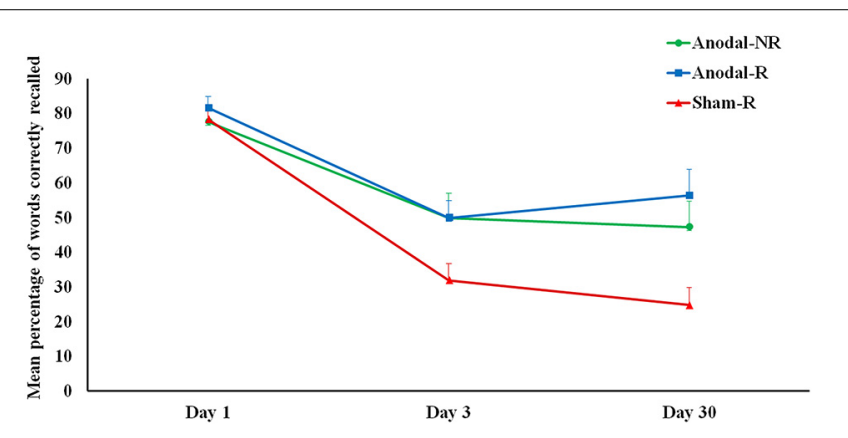

FIGURE 2 | The plot shows the mean percentage of words correctly recalled in each group (Anodal-R, Anodal-NR and Sham-R) at Day 1,

Day 3, and Day 30. There was significant memory decay (i.e., forgetting) from Day 1 to Day 3 in all groups. Forgetting was reduced up to 1 month in the anodal tDCS groups (Anodal-R and Anodal-NR) compared to the sham group (Sham-R). Error bars represent standard errors of the means (s.e.m.). $* p<0.05$.

Regarding the interaction, we found significant differences in all three groups between Day 1 and Day 3 (Anodal-NR Day 1 vs. Day $3 p<0.01$ ); (Anodal-R Day 1 vs. Day 3; $p<0.01$ ); (Sham$\mathrm{R}$ Day 1 vs. Day 3; $p<0.01$ ). Moreover, significant differences in all three groups between Day 1 and Day 30 (Anodal-NR Day 1 vs. Day 30; $p<0.01$ ); (Anodal-R Day 1 vs. Day 30; $p<0.01$ ); (Sham-R Day 1 vs. Day 30; $p<0.01$ ).

These findings show memory decay (i.e., forgetting) from Day 1 to Day 3 and from Day 1 to Day 30 in all groups. In addition, in the Sham- $\mathrm{R}$ group we observed a significant decrease in memory performance at Day 3 compared to Anodal-NR $(p=0.02)$ and Anodal-R $(p=0.02)$. Moreover, we observed a significant decrease of memory performance at Day 30 in Sham-R compared to Anodal-NR $(p<0.01)$ and Anodal-R $(p<0.01)$. These results show reduced forgetting up to 1 month in the anodal tDCS groups compared to the control group (Sham-R) (see Figure 2).

\section{DISCUSSION}

The results of this study show that anodal tDCS over the left DLPFC (i.e., Anodal-R and Anodal-NR groups) strengthened existing memories and reduced forgetting in healthy older subjects compared to the sham group (i.e., Sham-R). Importantly, this behavioral effect was not influenced by differences between groups in the learning rate, number of words correctly recalled in the last learning trial, cognitive reserve accumulated and memory strategies used.

Previous behavioral (Hupbach et al., 2008) and rTMS (Sandrini et al., 2013) reconsolidation studies in healthy young adults have shown that under laboratory conditions the original spatial context (i.e., same experimental room of Day 1) plays a role in reactivating the existing memories. In our study involving older adults, we observed similar behavioral facilitation effects (i.e., memory strengthening) in both the anodal tDCS over the left DLPFC groups, despite participants in the AnodalNR group were tested in a different room (i.e., different spatial context). Depending on subject population and features of the environment, memory reactivation might be triggered by other factors. Unlike the young adults (Sandrini et al., 2013), being in the same institute/center may have been more salient to older adults than the distinction between the two rooms, and the no reminder group (Anodal-NR) would have been reminded of the learning session and performed the same as the reminder group (Anodal-R). There is evidence that older adults have poor memory for the source and problems with the process of binding memories so that perceptual and contextual cues may be encoded but may not be appropriately bound to the target event (Schacter et al., 1991; Naveh-Benjamin and Craik, 1995; Chalfonte and Johnson, 1996). In addition, Kroes and colleagues using electroconvulsive therapy in patients with unipolar depression showed memory reconsolidation impairment, despite a change of room in the hospital, a finding that highlights the strength of the hospital context for allowing memory reactivation (Kroes et al., 2014). 
Regardless of spatial context, a previous reconsolidation study demonstrated that anodal tDCS over the left DLPFC strengthens verbal episodic memories in young subjects only when the existing memories have been reactivated (Javadi and Cheng, 2013). Although all these findings suggest that our facilitation effect observed in the both anodal tDCS groups might be triggered by the reactivation of the existing memories, further work is needed on the reconsolidation process in the elderly.

Considering the design limitations due to the fact that a sham-no reminder group was not tested, and the lack of statistical significance between Day 3 and Day 30 in all groups (see Figure 2), the behavioral effects observed at 1 month might be not due to the effect of anodal tDCS but to the repeated reactivation of the existing memories during testing (four free recall trials) on Day 3. Strengthening effects as a result of reconsolidation have been reported in animal and human studies with successive reactivations of the memories (Forcato et al., 2011; Inda et al., 2011). Inda et al. (2011), using inhibitory avoidance learning in rats, found that successive reactivations of existing memories, by re-exposition to the context, resulted in reconsolidation that mediated memory strengthening and prevents forgetting.

Previous studies have shown that anodal tDCS may enhance retrieval (Manenti et al., 2013) and consolidation (Floel et al., 2012) of episodic memories in the elderly. Manenti et al. (2013) demonstrated that anodal tDCS applied over the left DLPFC or left posterior parietal cortex during the retrieval phase enhanced recognition of verbal memories. In addition, Floel et al. (2012) showed that anodal tDCS over the right temporo-parietal cortex during an object-location task did not alter the learning rate and the immediate free recall but significantly enhanced the delayed recall (1 week) compared to sham (Floel et al., 2012). These findings further support the hypothesis (Reis et al., 2009) that there is a consolidation mechanism that is susceptible to anodal tDCS and contributes to offline effects more than to online effects.

Here, we show for the first time that anodal tDCS over the left DLPFC strengthens existing verbal episodic memories and reduces forgetting in the elderly. In addition, this study confirms the critical role of left DLPFC in verbal episodic memory along the lifespan (Gagnon et al., 2010; Manenti et al., 2010, 2011, 2013; Gagnon et al., 2011; Javadi and Walsh, 2012; Javadi and Cheng, 2013).

Regarding the putative brain mechanisms of this facilitation effect, there is evidence that tDCS affects not only the targeted local region but also activity in remote interconnected regions (Pena-Gomez et al., 2012; Venkatakrishnan and Sandrini, 2012; Saiote et al., 2013; Stagg et al., 2013). Anodal tDCS over the left DLPFC might have enhanced the functional coupling between the PFC and the hippocampus, thereby enhancing memory recollection. This speculation has also been suggested in a similar reconsolidation study using $1 \mathrm{~Hz}$ rTMS over the right DLPFC in young subjects (Sandrini et al., 2013). This might initially seem counterintuitive given the widely used rule of thumb that $1 \mathrm{~Hz}$ stimulation decreases cortical excitability, inducing inhibitory effects. However, this principle is mainly derived from basic studies of motor cortex and does not necessarily apply to other cortical regions and more complex cognitive functions (Sandrini et al., 2011). For instance, Turriziani et al. (2012) showed that
$1 \mathrm{~Hz}$ rTMS (considered to have an inhibitory effect) of right DLPFC enhanced episodic memory while intermittent Theta Burst Stimulation (considered to have a facilitatory effect) of the same region deteriorated memory performance. In addition, there is evidence that $1 \mathrm{~Hz}$ rTMS may improve performance of a cognitive task by strengthening the connectivity between taskrelevant brain regions depending on the functional state of the cortex at the time of stimulation (Ward et al., 2010).

Combined NIBS and neuroimaging studies (Censor et al., 2013, 2014; Macher et al., 2014; Vidal-Pineiro et al., 2014) may shed light on how functional interactions between remote but interconnected brain regions may mediate strengthening of existing memories in young and older adults.

In conclusion, anodal tDCS over the left DLPFC induces beneficial effects on verbal episodic memories in older adults, suggesting that noninvasive stimulation of this cortical region might be a novel strategy to strengthen existing memories and reduce memory loss in older adults with episodic memory impairment, such as aMCI.

\section{ACKNOWLEDGMENTS}

This work was partially supported by funding from United States Department of Defense in the Center for Neuroscience and Regenerative Medicine (CNRM) to Marco Sandrini. The authors are indebted with Clarissa Ferrari for assistance in the statistical analysis.

\section{SUPPLEMENTARY MATERIAL}

The Supplementary Material for this article can be found online at: http://www.frontiersin.org/journal/10.3389/fnagi.2014. 00289/abstract

\section{REFERENCES}

Alberini, C. M., and Chen, D. Y. (2012). Memory enhancement: consolidation, reconsolidation and insulin-like growth factor 2. Trends Neurosci. 35, 274-283. doi: 10.1016/j.tins.2011.12.007

Alberini, C. M., and Ledoux, J. E. (2013). Memory reconsolidation. Curr. Biol. 23, R746-R750. doi: 10.1016/j.cub.2013.06.046

Bos, M. G., Schuijer, J., Lodestijn, F., Beckers, T., and Kindt, M. (2014). Stress enhances reconsolidation of declarative memory. Psychoneuroendocrinology 46, 102-113. doi: 10.1016/j.psyneuen.2014.04.011

Brem, A. K., Ran, K., and Pascual-Leone, A. (2013). Learning and memory. Handb. Clin. Neurol. 116, 693-737. doi: 10.1016/B978-0-444-53497-2.00055-3

Buschert, V., Bokde, A. L., and Hampel, H. (2011). Cognitive intervention in Alzheimer disease. Nat. Rev. Neurol. 6, 508-517. doi: 10.1038/nrneurol.2010.113

Cabeza, R., and Lennartson, E. R. (2005). False memory across languages: implicit associative response vs fuzzy trace views. Memory 13, 1-5. doi: 10.1080/09658210344000161

Censor, N., Dayan, E., and Cohen, L. G. (2013). Cortico-subcortical neuronal circuitry associated with reconsolidation of human procedural memories. Cortex 8, 281-288. doi: 10.1016/j.cortex.2013.05.013

Censor, N., Dimyan, M. A., and Cohen, L. G. (2010). Modification of existing human motor memories is enabled by primary cortical processing during memory reactivation. Curr. Biol. 20, 1545-1549. doi: 10.1016/j.cub.2010. 07.047

Censor, N., Horovitz, S. G., and Cohen, L. G. (2014). Interference with existing memories alters offline intrinsic functional brain connectivity. Neuron 81 , 69-76. doi: 10.1016/j.neuron.2013.10.042

Chalfonte, B. L., and Johnson, M. K. (1996). Feature memory and binding in young and older adults. Mem. Cogn. 24, 403-416. doi: 10.3758/BF03200930

Chan, J. C., and LaPaglia, J. A. (2013). Impairing existing declarative memory in humans by disrupting reconsolidation. Proc. Natl. Acad. Sci. U.S.A. 110, 9309-9313. doi: 10.1073/pnas.1218472110 
Coccoz, V., Maldonado, H., and Delorenzi, A. (2011). The enhancement of reconsolidation with a naturalistic mild stressor improves the expression of a declarative memory in humans. Neuroscience 185, 61-72. doi: 10.1016/j.neuroscience.2011.04.023

Coccoz, V., Sandoval, A. V., Stehberg, J., and Delorenzi, A. (2013). The temporal dynamics of enhancing a human declarative memory during reconsolidation. Neuroscience 246, 397-408. doi: 10.1016/j.neuroscience.2013.04.033

Cotelli, M., Manenti, R., Zanetti, O., and Miniussi, C. (2012). Nonpharmacological intervention for memory decline. Front. Hum. Neurosci. 6:46. doi: 10.3389/fnhum.2012.00046

Daselaar, S. M., and Cabeza, R. (2008). "Episodic memory Decline and Healthy Aging: Role of Prefrontal and Medial Temporal Lobe Regions," in Learning and Memory: a Comprehensive Reference, ed J. H. Byrne (Oxford, UK: Elsevier), 577-599.

Dayan, E., Censor, N., Buch, E. R., Sandrini, M., and Cohen, L. G. (2013). Noninvasive brain stimulation: from physiology to network dynamics and back. Nat. Neurosci. 16, 838-844. doi: 10.1038/nn.3422

De Renzi, E., Motti, F., and Nichelli, P. (1980). Imitating gestures. A quantitative approach to ideomotor apraxia. Arch. Neurol. 37, 6-10. doi: 10.1001/archneur. 1980.00500500036003

Dudai, Y. (2012). The restless engram: consolidations never end. Annu. Rev. Neurosci. 35, 227-247. doi: 10.1146/annurev-neuro-062111-150500

Fertonani, A., Rosini, S., Cotelli, M., Rossini, P. M., and Miniussi, C. (2010). Naming facilitation induced by transcranial direct current stimulation. Behav. Brain Res. 208, 311-318. doi: 10.1016/j.bbr.2009.10.030

Finn, B., and Roediger, H. L. 3rd. (2011). Enhancing retention through reconsolidation: negative emotional arousal following retrieval enhances later recall. Psychol. Sci. 22, 781-786. doi: 10.1177/0956797611407932

Fletcher, P. C., and Henson, R. N. (2001). Frontal lobes and human memory: insights from functional neuroimaging. Brain 124, 849-881. doi: 10.1093/brain/124.5.849

Floel, A., Suttorp, W., Kohl, O., Kurten, J., Lohmann, H., Breitenstein, C., et al. (2012). Non-invasive brain stimulation improves objectlocation learning in the elderly. Neurobiol. Aging 33, 1682-1689. doi: 10.1016/j.neurobiolaging.2011.05.007

Folstein, M. F., Folstein, S. E., and McHugh, P. R. (1975). "Mini-mental state." A practical method for grading the cognitive state of patients for the clinician. J. Psychiatr. Res. 12, 189-198. doi: 10.1016/0022-3956(75)90026-6

Forcato, C., Burgos, V. L., Argibay, P. F., Molina, V. A., Pedreira, M. E., and Maldonado, H. (2007). Reconsolidation of declarative memory in humans. Learn. Mem. 14, 295-303. doi: 10.1101/lm.486107

Forcato, C., Rodriguez, M. L., and Pedreira, M. E. (2011). Repeated labilizationreconsolidation processes strengthen declarative memory in humans. PLoS ONE 6:e23305. doi: 10.1371/journal.pone.0023305

Gagnon, G., Blanchet, S., Grondin, S., and Schneider, C. (2010). Paired-pulse transcranial magnetic stimulation over the dorsolateral prefrontal cortex interferes with episodic encoding and retrieval for both verbal and non-verbal materials. Brain Res. 1344, 148-158. doi: 10.1016/j.brainres.2010.04.041

Gagnon, G., Schneider, C., Grondin, S., and Blanchet, S. (2011). Enhancemen of episodic memory in young and healthy adults: a paired-pulse TMS study on encoding and retrieval performance. Neurosci. Lett. 488, 138-142. doi: 10.1016/j.neulet.2010.11.016

Gandiga, P. C., Hummel, F. C., and Cohen, L. G. (2006). Transcranial DC stimulation (tDCS): a tool for double-blind sham-controlled clinical studies in brain stimulation. Clin. Neurophysiol. 117, 845-850. doi: 10.1016/j.clinph.2005.12.003

Grady, C. L., and Craik, F. I. (2000). Changes in memory processing with age. Curr. Opin. Neurobiol. 10, 224-231. doi: 10.1016/S0959-4388(00)00073-8

Hayter, A. J. (1986). The maximum familywise error rate of Fisher's Least Significant Difference Test. J. Am. Stat. Assoc. 81, 1000-1004. doi: 10.1080/01621459.1986.10478364

Herwig, U., Satrapi, P., and Schonfeldt-Lecuona, C. (2003). Using the international 10-20 EEG system for positioning of transcranial magnetic stimulation. Brain Topogr. 16, 95-99. doi: 10.1023/B:BRAT.0000006333.93597.9d

Hupbach, A., Gomez, R., Hardt, O., and Nadel, L. (2007). Reconsolidation of episodic memories: a subtle reminder triggers integration of new information. Learn. Mem. 14, 47-53. doi: 10.1101/lm.365707

Hupbach, A., Gomez, R., and Nadel, L. (2009). Episodic memory reconsolidation: updating or source confusion? Memory 17, 502-510. doi: $10.1080 / 09658210902882399$
Hupbach, A., Hardt, O., Gomez, R., and Nadel, L. (2008). The dynamics of memory: context-dependent updating. Learn. Mem. 15, 574-579. doi: 10.1101/lm.1022308

Inda, M. C., Muravieva, E. V., and Alberini, C. M. (2011). Memory retrieval and the passage of time: from reconsolidation and strengthening to extinction. J. Neurosci. 31, 1635-1643. doi: 10.1523/JNEUROSCI.473610.2011

Javadi, A. H., and Cheng, P. (2013). Transcranial direct current stimulation (tDCS) enhances reconsolidation of long-term memory. Brain Stimul. 6, 668-674. doi: 10.1016/j.brs.2012.10.007

Javadi, A. H., and Walsh, V. (2012). Transcranial direct current stimulation (tDCS) of the left dorsolateral prefrontal cortex modulates declarative memory. Brain Stimul. 5, 231-241. doi: 10.1016/j.brs.2011.06.007

Kroes, M. C., Tendolkar, I., van Wingen, G. A., van Waarde, J. A., Strange, B. A., and Fernandez, G. (2014). An electroconvulsive therapy procedure impairs reconsolidation of episodic memories in humans. Nat. Neurosci. 17, 204-206. doi: 10.1038/nn.3609

Laudanna, A., Thornton, A. M., Brown, G., Burani, C., and Marconi, L. (1995). "Un corpus dell'italiano scritto contemporaneo dalla parte del ricevente," in III Giornate Internazionali di Analisi Statistica dei Dati Testuali, Vol. 1, eds S. Bolasco, L. Lebart, and A. Salem (Roma: Cisu), 103-109.

Lezak, M., Howieson, D., and Loring, D. W. (2004). Neuropsychological Assessment, 4 Edn. Oxford: University Press.

Macher, K., Bohringer, A., Villringer, A., and Pleger, B. (2014). Cerebellar-parietal connections underpin phonological storage. J. Neurosci. 34, 5029-5037. doi: 10.1523/JNEUROSCI.0106-14.2014

Manenti, R., Brambilla, M., Petesi, M., Ferrari, C., and Cotelli, M. (2013). Enhancing verbal episodic memory in older and young subjects after non-invasive brain stimulation. Front. Aging Neurosci. 5:49. doi: 10.3389/fnagi.2013.00049

Manenti, R., Cotelli, M., and Miniussi, C. (2011). Successful physiological aging and episodic memory: a brain stimulation study. Behav. Brain Res. 216, 153-158. doi: 10.1016/j.bbr.2010.07.027

Manenti, R., Cotelli, M., Robertson, I. H., and Miniussi, C. (2012). Transcranial brain stimulation studies of episodic memory in young adults, elderly adults and individuals with memory dysfunction: a review. Brain Stimul. 5, 103-109. doi: 10.1016/j.brs.2012.03.004

Manenti, R., Tettamanti, M., Cotelli, M., Miniussi, C., and Cappa, S. F. (2010) The neural bases of word encoding and retrieval: a fMRI-guided transcranial magnetic stimulation study. Brain Topogr. 22, 318-332. doi: 10.1007/s10548009-0126-1

McGaugh, J. L. (2000). Memory-a century of consolidation. Science 287, 248-251. doi: $10.1126 /$ science. 287.5451 .248

Monfils, M. H., Cowansage, K. K., Klann, E., and LeDoux, J. E. (2009). Extinctionreconsolidation boundaries: key to persistent attenuation of fear memories. Science 324, 951-955. doi: 10.1126/science.1167975

Nadel, L., Hupbach, A., Gomez, R., and Newman-Smith, K. (2012). Memory formation, consolidation and transformation. Neurosci. Biobehav. Rev. 36, 1640-1645. doi: 10.1016/j.neubiorev.2012.03.001

Nader, K., and Hardt, O. (2009). A single standard for memory: the case for reconsolidation. Nat. Rev. Neurosci. 10, 224-234. doi: 10.1038/ $\operatorname{nrn} 2590$

Nader, K., Schafe, G. E., and Le Doux, J. E. (2000a). Fear memories require protein synthesis in the amygdala for reconsolidation after retrieval. Nature 406, 722-726. doi: 10.1038/35021052

Nader, K., Schafe, G. E., and LeDoux, J. E. (2000b). The labile nature of consolidation theory. Nat. Rev. Neurosci. 1, 216-219. doi: 10.1038/35044580

Naveh-Benjamin, M., and Craik, F. I. (1995). Memory for context and its use in item memory: comparisons of younger and older persons. Psychol. Aging 10, 284-293. doi: 10.1037/0882-7974.10.2.284

Nitsche, M. A., Cohen, L. G., Wassermann, E. M., Priori, A., Lang, N., Antal, A., et al. (2008). Transcranial direct current stimulation: state of the art 2008. Brain Stimul. 1, 206-223. doi: 10.1016/j.brs.2008.06.004

Nitsche, M. A., and Paulus, W. (2000). Excitability changes induced in the human motor cortex by weak transcranial direct current stimulation. J. Physiol. 527( $\mathrm{Pt}$ 3), 633-639. doi: 10.1111/j.1469-7793.2000.t01-1-00633.x

Nucci, M., Mapelli, D., and Mondini, S. (2012). Cognitive Reserve Index questionnaire (CRIq): a new instrument for measuring cognitive reserve. Aging Clin. Exp. Res. 24, 218-226. doi: 10.3275/7800 
Nyberg, L., Lovden, M., Riklund, K., Lindenberger, U., and Backman, L. (2012). Memory aging and brain maintenance. Trends Cogn. Sci. 16, 292-305. doi: 10.1016/j.tics.2012.04.005

Pena-Gomez, C., Sala-Lonch, R., Junque, C., Clemente, I. C., Vidal, D., Bargallo, N., et al. (2012). Modulation of large-scale brain networks by transcranial direct current stimulation evidenced by resting-state functional MRI. Brain Stimul. 5, 252-263. doi: 10.1016/j.brs.2011.08.006

Poreisz, C., Boros, K., Antal, A., and Paulus, W. (2007). Safety aspects of transcranial direct current stimulation concerning healthy subjects and patients. Brain Res. Bull. 72, 208-214. doi: 10.1016/j.brainresbull.2007.01.004

Reis, J., Schambra, H. M., Cohen, L. G., Buch, E. R., Fritsch, B., Zarahn, E., et al. (2009). Noninvasive cortical stimulation enhances motor skill acquisition over multiple days through an effect on consolidation. Proc. Natl. Acad. Sci. U.S.A. 106, 1590-1595. doi: 10.1073/pnas.0805413106

Rodriguez, M. L., Campos, J., Forcato, C., Leiguarda, R., Maldonado, H., Molina, V. A., et al. (2013). Enhancing a declarative memory in humans: the effect of clonazepam on reconsolidation. Neuropharmacology 64, 432-442. doi: 10.1016/j.neuropharm.2012.06.059

Ronnlund, M., Nyberg, L., Backman, L., and Nilsson, L. G. (2005). Stability, growth, and decline in adult life span development of declarative memory: cross-sectional and longitudinal data from a population-based study. Psychol. Aging 20, 3-18. doi: 10.1037/0882-7974.20.1.3

Rossi, S., Cappa, S. F., Babiloni, C., Pasqualetti, P., Miniussi, C., Carducci, F., et al. (2001). Prefrontal cortex in long-term memory: an "interference" approach using magnetic stimulation. Nat. Neurosci. 4, 948-952. doi: 10.1038/ nn0901-948

Rossi, S., Miniussi, C., Pasqualetti, P., Babiloni, C., Rossini, P. M., and Cappa, S. F. (2004). Age-related functional changes of prefrontal cortex in long-term memory: a repetitive transcranial magnetic stimulation study. J. Neurosci. 24, 7939-7944. doi: 10.1523/JNEUROSCI.0703-04.2004

Saiote, C., Turi, Z., Paulus, W., and Antal, A. (2013). Combining functional magnetic resonance imaging with transcranial electrical stimulation. Front. Hum. Neurosci. 7:435. doi: 10.3389/fnhum.2013.00435

Sala-Llonch, R., Junque, C., Arenaza-Urquijo, E. M., Vidal-Pineiro, D., Valls-Pedret, C., Palacios, E. M., et al. (2014). Changes in whole-brain functional networks and memory performance in aging. Neurobiol. Aging 35, 2193-2202. doi: 10.1016/j.neurobiolaging.2014.04.007

Sandrini, M., Cappa, S. F., Rossi, S., Rossini, P. M., and Miniussi, C. (2003). The role of prefrontal cortex in verbal episodic memory: rTMS evidence. J. Cogn. Neurosci. 15, 855-861. doi: 10.1162/089892903322370771

Sandrini, M., Censor, N., Mishoe, J., and Cohen, L. G. (2013). Causal role of prefrontal cortex in strengthening of episodic memories through reconsolidation. Curr. Biol. 23, 2181-2184. doi: 10.1016/j.cub.2013.08.045

Sandrini, M., and Cohen, L. G. (2013). Noninvasive brain stimulation in neurorehabilitation. Handb. Clin. Neurol. 116, 499-524. doi: 10.1016/B978-0-44453497

Sandrini, M., and Cohen, L. G. (2014). "Effects of brain stimulation on declarative and procedural memories," The Stimulated Brain, Chapter 9, ed R. CohenKadosh (Elsevier), 237-256.

Sandrini, M., Umilta, C., and Rusconi, E. (2011). The use of transcranial magnetic stimulation in cognitive neuroscience: a new synthesis of methodological issues. Neurosci. Biobehav. Rev. 35, 516-536. doi: 10.1016/j.neubiorev.2010.06.005

Schacter, D. L., Kaszniak, A. W., Kihlstrom, J. F., and Valdiserri, M. (1991). The relation between source memory and aging. Psychol. Aging 6, 559-568. doi: 10.1037/0882-7974.6.4.559

Schwabe, L., Nader, K., and Pruessner, J. C. (2013). $\beta$-Adrenergic blockade during reactivation reduces the subjective feeling of remembering associated with emotional episodic memories. Biol. Psychol. 92, 227-232. doi: 10.1016/j.biopsycho.2012.10.003

Schwabe, L., Nader, K., and Pruessner, J. C. (2014). Reconsolidation of human memory: brain mechanisms and clinical relevance. Biol. Psychiatry 76, 274-280. doi: 10.1016/j.biopsych.2014.03.008

Simons, J. S., and Spiers, H. J. (2003). Prefrontal and medial temporal lobe interactions in long-term memory. Nat. Rev. Neurosci. 4, 637-648. doi: $10.1038 / \mathrm{nrn} 1178$

Stagg, C. J., Lin, R. L., Mezue, M., Segerdahl, A., Kong, Y., Xie, J., et al. (2013). Widespread modulation of cerebral perfusion induced during and after transcranial direct current stimulation applied to the left dorsolateral prefrontal cortex. J. Neurosci. 33, 11425-11431. doi: 10.1523/JNEUROSCI.3887-12.2013

Strange, B. A., Kroes, M. C., Fan, J. E., and Dolan, R. J. (2010). Emotion causes targeted forgetting of established memories. Front. Behav. Neurosci. 4:175. doi: 10.3389/fnbeh.2010.00175

Tulving, E. (1983). Elements of Episodic Memory. London: Oxford UP.

Turriziani, P., Smirni, D., Zappala, G., Mangano, G. R., Oliveri, M., and Cipolotti, L. (2012). Enhancing memory performance with rTMS in healthy subjects and individuals with Mild Cognitive Impairment: the role of the right dorsolateral prefrontal cortex. Front. Hum. Neurosci. 6:62. doi: 10.3389/fnhum.2012.00062

Venkatakrishnan, A., and Sandrini, M. (2012). Combining transcranial direct current stimulation and neuroimaging: novel insights in understanding neuroplasticity. J. Neurophysiol. 107, 1-4. doi: 10.1152/jn.00557.2011

Vestergren, P., and Nilsson, L. G. (2010). Perceived causes of everyday memory problems in a population-based sample aged 39-99. Appl. Cogn. Psychol. 25, 641-646. doi: 10.1002/acp.1734

Vidal-Pineiro, D., Martin-Trias, P., Arenaza-Urquijo, E. M., Sala-Llonch, R., Clemente, I. C., Mena-Sanchez, I., et al. (2014). Task-dependent activity and connectivity predict episodic memory network-based responses to brain stimulation in healthy aging. Brain Stimul. 7, 287-296. doi: 10.1016/j.brs.2013.12.016

Ward, N. S., Bestmann, S., Hartwigsen, G., Weiss, M. M., Christensen, L. O., Frackowiak, R. S., et al. (2010). Low-frequency transcranial magnetic stimulation over left dorsal premotor cortex improves the dynamic control of visuospatially cued actions. J. Neurosci. 30, 9216-9223. doi: 10.1523/JNEUROSCI.449909.2010

Zimerman, M., and Hummel, F. C. (2010). Non-invasive brain stimulation: enhancing motor and cognitive functions in healthy old subjects. Front. Aging Neurosci. 2:149. doi: 10.3389/fnagi.2010.00149

Conflict of Interest Statement: The authors declare that the research was conducted in the absence of any commercial or financial relationships that could be construed as a potential conflict of interest.

Received: 15 July 2014; accepted: 30 September 2014; published online: 20 October 2014.

Citation: Sandrini M, Brambilla M, Manenti R, Rosini S, Cohen LG and Cotelli $M$ (2014) Noninvasive stimulation of prefrontal cortex strengthens existing episodic memories and reduces forgetting in the elderly. Front. Aging Neurosci. 6:289. doi: 10.3389/fnagi.2014.00289

This article was submitted to the journal Frontiers in Aging Neuroscience.

Copyright (c) 2014 Sandrini, Brambilla, Manenti, Rosini, Cohen and Cotelli. This is an open-access article distributed under the terms of the Creative Commons Attribution License (CC BY). The use, distribution or reproduction in other forums is permitted, provided the original author (s) or licensor are credited and that the original publication in this journal is cited, in accordance with accepted academic practice. No use, distribution or reproduction is permitted which does not comply with these terms. 